\title{
A CAMERON-MARTIN TYPE QUASI-INVARIANCE THEOREM FOR PINNED BROWNIAN MOTION ON A COMPACT RIEMANNIAN MANIFOLD
}

\author{
BRUCE K. DRIVER
}

\begin{abstract}
The results in Driver [13] for quasi-invariance of Wiener measure on the path space of a compact Riemannian manifold $(M)$ are extended to the case of pinned Wiener measure. To be more explicit, let $h:[0,1] \rightarrow T_{o} M$ be a $C^{1}$ function where $M$ is a compact Riemannian manifold, $o \in M$ is a base point, and $T_{o} M$ is the tangent space to $M$ at $o \in M$. Let $W(M)$ be the space of continuous paths from $[0,1]$ into $M, \nu$ be Wiener measure on $W(M)$ concentrated on paths starting at $o \in M$, and $H_{s}(\omega)$ denote the stochastic-parallel translation operator along a path $\omega \in W(M)$ up to "time" s. (Note: $H_{s}(\omega)$ is only well defined up to $\nu$-equivalence.) For $\omega \in W(M)$ let $X^{h}(\omega)$ denote the vector field along $\omega$ given by $X_{s}^{h}(\omega) \equiv H_{s}(\omega) h(s)$ for each $s \in[0,1]$. One should interpret $X^{h}$ as a vector field on $W(M)$. The vector field $X^{h}$ induces a flow $S^{h}(t, \cdot): W(M) \rightarrow W(M)$ which leaves Wiener measure $(\nu)$ quasi-invariant, see Driver [13]. It is shown in this paper that the same result is valid if $h(1)=0$ and the Wiener measure $(\nu)$ is replaced by a pinned Wiener measure $\left(\nu_{e}\right)$. (The measure $\nu_{e}$ is proportional to the measure $\nu$ conditioned on the set of paths which start at $o \in M$ and end at a fixed end point $e \in M$.) Also as in [13], one gets an integration by parts formula for the vector-fields $X^{h}$ defined above.
\end{abstract}

\section{INTRODUCTION}

Let $\mu$ denote standard Wiener measure on $W\left(\mathbb{R}^{d}\right) \equiv C\left([0,1], \mathbb{R}^{d}\right)$, and $\mathbf{H}$ denote the set of $h \in W\left(\mathbb{R}^{d}\right)$ such that $h$ is $C^{1}$ and $h(0)=0$. The classical Cameron-Martin theorem states (see Cameron and Martin [7]) that $\mu^{h} \equiv \mu(\cdot-h)$ is equivalent to $\mu$ and the Radon-Nikodym derivative of $\mu^{h}$ with respect to $\mu$ is

$$
\frac{d \mu^{h}}{d \mu}(\omega)=\exp \left\{\int_{0}^{1} h(s) \cdot d \omega(s)-\frac{1}{2} \int_{0}^{1}|h(s)|^{2} d s\right\} .
$$

There has since been many extensions to this theorem, see for example Cameron and Martin [8, 9], Girsanov [18], Gross [19], Kuo [27, 28], Kusuoka [30-33], and Ramer [45]. There is also the group of extensions of the Cameron-Martin theorem used in the study of loop groups; see Albeverio and Hoegh-Krohn [2], Frenkel [16], Gross [22], and Malliavin and Malliavin [35]. In Driver

Received by the editors March 12, 1992.

1991 Mathematics Subject Classification. Primary 60D05, 28D05; Secondary 58D20, 58D25.

This research was partially supported by NSF Grant No. DMS 91-01720. 
[13] it is shown that the classical Cameron-Martin theorem extends to the case of compact Riemannian manifolds (see Theorem 3.1), which includes Wiener measure on the path space $W(G)$ of a compact Lie group $G$. The purpose of this paper is to extend the results in [13] to the case of "pinned Wiener measure" on a compact Riemannian manifold $M$; see Proposition 3.3 and Theorem 3.4. We also derive an integration by parts formula for " $h$-derivatives"; see Theorem 3.13 .

\section{NOTATION AND BASIC PROPERTIES OF PINNED BROWNIAN MOTION}

In this section we setup some notation and review a number of prerequisites for this article. The reader may also wish to consult Driver [13] for a more detailed exposition of some of the material in this section.

2.1. Differential geometric preliminaries. In this paper the data $\left(M^{d}, g, \nabla, o\right.$, $e, u_{o}$ ) will be fixed, where $M$ is a compact connected manifold (without boundary) of dimension $d, g$ is a Riemannian metric on $M, \nabla$ is a $g$-compatible covariant derivative, $o$ and $e$ are fixed points in $M$, and $u_{o}$ is an orthonormal frame at $o \in M$. (We view $u_{o}$ as an isometry from $\mathbb{R}^{d}$ to $T_{o} M$, the tangent space to $M$ at $o$.) The symbol $\nabla$ will also be used to denote the the gradient operator acting on functions on $M$. The orthogonal frame bundle over $M$ will be denoted by $O(M)$ and $\pi: O(M) \rightarrow M$ will be the fiber projection map. Throughout this paper the covariant derivative $(\nabla)$ is always assumed to be $g$-compatible and "torsion skew symmetric" or TSS for short. The connection $(\nabla)$ is said to be torsion skew symmetric if the torsion tensor $(T)$ satisfies $g(T(v, w), w)=0$ for all $v, w \in T_{m} M$ and $m \in M$.

The Laplacian $(\Delta)$ with respect to $(\nabla)$ is the second order differential operator acting on the smooth functions $f \in C^{\infty}(M)$ given by $\Delta f \equiv s p \nabla d f \equiv$ $\sum_{i=1}^{n}\left\{E_{i} E_{i} f-d f\left\langle\nabla_{E_{i}} E_{i}\right\rangle\right\}$, where $\left\{E_{i}\right\}_{i=1}^{n}$ is a local orthonormal frame. We recall from Driver [13] that this Laplacian is the same as the Levi-Civita Laplacian due to TSS condition on $(\nabla)$.

The Riemannian distance on $M$ will be denoted by $d(\cdot, \cdot)$. That is for $x, y \in M, d(x, y) \equiv \inf _{\sigma} \int_{0}^{1}\left|\sigma^{\prime}(s)\right| d s$, where the infimum is taken over all $C^{1}$-paths $\sigma:[0,1] \rightarrow M$ such that $\sigma(0)=x$ and $\sigma(1)=y$. I have also written $\left|\sigma^{\prime}(s)\right|$ for $\left(g\left\langle\sigma^{\prime}(s), \sigma^{\prime}(s)\right\rangle\right)^{1 / 2}$.

The standard horizontal vector fields on $O(M)$ will be denoted by $\{B\langle a\rangle\}_{a \in \mathbb{R}^{d}}$ -recall that $B\langle a\rangle(u)$ is by definition the horizontal lift of the tangent vector $u a \in T_{\pi(u)} M$ to $T_{u} O(M)$. The fundamental 1 -form on $O(M)$ is the $\mathbb{R}^{d}$-valued 1-form $(\theta)$ on $O(M)$ given by $\theta\left\langle u^{\prime}\right\rangle=u^{-1} \pi_{*} u^{\prime}$, where $u(s)$ is a smooth path in $O(M)$. The connection 1 -form is the $s o(n)$-valued 1 -form on $O(M)$ defined by $\omega\left\langle u^{\prime}\right\rangle \equiv u^{-1} \nabla u / d s$, where $\nabla u / d s$ denotes the covariant derivative of $u$ along $\sigma \equiv \pi \circ u$. (Here $\nabla u(s) / d s: \mathbb{R}^{d} \rightarrow T_{\pi(u(s))} M$ and $\nabla u(s) a / d s \equiv \nabla(u(s) a) / d s$ for all $a \in \mathbb{R}^{d}$.)

It will also be convenient to define the "equivariant" form of the curvature and torsion tensors. These forms are given by $\Omega_{u}\langle a, b\rangle \equiv u^{-1} R\langle u a, u b\rangle u \in$ $s o(n)$, and $\Theta_{u}\langle a, b\rangle \equiv u^{-1} T\langle u a, u b\rangle \in \mathbb{R}^{d}$, where $R$ is the curvature tensor of $(\nabla), \quad T$ is the torsion tensor, $u \in O(M)$, and $a$ and $b$ are in $\mathbb{R}^{d}$. For more details on this notation see Driver [13] and Kobayashi and Nomizu [26]. 
2.2. Heat kernel estimates. This section collects a number of estimates involving the heat kernel which will be needed in the sequel. Let $p_{s}(x, y)$ be the kernel (with respect to the Riemannian volume form on $M$ ) of the heat operator $e^{s \Delta / 2}$. Alternatively, $p_{s}(x, y)$ is the value at $x \in M$ of the fundamental solution to the heat equation $\partial f / \partial s=\frac{1}{2} \Delta f$ with initial condition $f^{\prime}(0, \cdot)=\delta_{y}(\cdot)$. We summarize the estimates that will be used in this paper in the following theorem.

Theorem 2.1. There are constants $\epsilon>0, K>0$, and $C_{i}>0$ for $i=1, \ldots, 4$ such that the following estimates hold for all $x, y$, and $e \in M$, and $0<s \leq 1$ :

$$
\begin{gathered}
\epsilon s^{-d / 2} e^{-C_{1} d(x, e)^{2} / s} \leq\left|p_{s}(x, e)\right| \leq K s^{-d / 2} e^{-C_{2} d(x, e)^{2} / s}, \\
\left|\nabla_{x} \ln p_{s}(x, e)\right| \leq C_{3}[d(x, e) / s+1 / \sqrt{s}], \\
0<p_{s}(x, e) / p_{s}(y, e) \leq e^{C_{4} d(x, y) / s} .
\end{gathered}
$$

Furthermore, the kernel $p_{s}(x, e)$ has the asymptotic expansion valid for $x$ near $e$ and $s$ near zero:

$$
p_{s}(x, e)=(2 \pi s)^{-1} e^{-d(x, e)^{2} / 2 s}\{1+O(s)\} .
$$

Proof. The estimates in (2.1) are well known and can be found in a number of references, see for example, Li and Yau [34], Cheeger and Yau [10], Davies [11], Hamilton [23], Setti [49], and Varopoulos [54, 55]. Probabilistic expressions for the left member of (2.2) may be found for example in Bismut [4], Jones and Leandre [25], and Norris [43].

For the purposes of this paper the author has found the Hamilton paper most useful. Equation (2.2) is an easy consequence of the results in [23] and equation (2.1). Indeed, Corollary 1.3 in [23] asserts that there are constants $A$ and $B$ depending only on $(M, g)$ such that

$$
s\left|\nabla \log p_{s}(x, e)\right|^{2} \leq A \log \left(B / s^{d / 2} p_{s}(x, e)\right) .
$$

It is now trivial to conclude (2.2) from this estimate and the lower bound in (2.1).

To prove (2.3) let $\sigma:[0,1] \rightarrow M$ be a smooth path such that $\sigma(0)=y$ and $\sigma(1)=x$. To simplify notation let $l(t) \equiv \log p_{s}(\sigma(t), e)$. Then it follows from (2.2) (with $C_{4}$ sufficiently large) that

$$
|l(1)-l(0)| \leq \int_{0}^{1}\left|\nabla \log p_{s}(\sigma(t), e) \cdot \dot{\sigma}(t)\right| d t \leq \frac{C_{4}}{s} \int_{0}^{1}|\dot{\sigma}(t)| d t,
$$

where we have used the fact that $d(\cdot, e)$ is bounded since $M$ is compact. Now take the infimum of the right-hand member of this last inequality to find that

$$
\left|\log \left(p_{s}(x, e) / p_{s}(y, e)\right)\right|=\left|\log p_{s}(x, e)-\log p_{s}(y, e)\right| \leq \frac{C_{4}}{s} d(x, y) .
$$

Equation (2.3) now easily follows.

Finally the asymptotic expansion in (2.4) is well known and can be found in a number of references; see for example $[18,47]$. 
2.3. Brownian motions and pinned Brownian motions. We start this section with some measure theoretic notation and conventions. Suppose that $\left(\Omega,\left\{\mathscr{F}_{s}\right\}, \mathscr{F}\right)$ is a filtered measurable space, $P$ and $Q$ are measures on $(\Omega, \mathscr{F})$, and $f$ : $\Omega \rightarrow \mathbb{R}$ is a $\mathscr{F}$-measurable function. The measures $P$ and $Q$ are said to be equivalent if $P$ and $Q$ are absolutely continuous with respect to one another. If $\rho=d Q / d P$ is the Radon-Nikodym derivative of of $Q$ with respect to $P$ we will write $Q=\rho \cdot P$. The integral $\int_{\Omega} f(\omega) d P(\omega)$ will often be denoted simply by $P(f)$. More generally if $\mathscr{H} \subset \mathscr{F}$ is a sub-sigma field of $\mathscr{F}$ then $P(f \mid \mathscr{H})$ will denote the conditional expectation of $f$ relative to $\mathscr{H}$ and $P$.

Remark 2.2. We will find it convenient to work with filtered measure spaces $\left(\Omega,\left\{\mathscr{F}_{s}\right\}, \mathscr{F}, P\right)$ where $P$ is a finite but not necessarily a probability measure. In these cases we say that a process $\left\{X_{s}\right\}$ is an $\left(\Omega,\left\{\mathscr{F}_{s}\right\}, \mathscr{F}, P\right)$-martingale or Brownian motion if $\left\{X_{s}\right\}$ is an $\left(\Omega,\left\{\mathscr{F}_{s}\right\}, \mathscr{F}, P(\Omega)^{-1} P\right)$-martingale or Brownian motion respectively.

Suppose that $\mathrm{Q}$ is any manifold and that $q_{o} \in Q$ is a fixed base point. Let $W(Q) \equiv C([0,1], Q)$ be the set of continuous paths from $[0,1]$ to $Q$. Let $W_{q_{o}}(Q)$ be the subset of paths in $W(Q)$ which start at $q_{o}$. Given a function $\phi: \Omega \rightarrow W(Q)$ and $s \in[0,1]$, let $\phi_{s}: \Omega \rightarrow Q$ be defined by $\phi_{s}(\omega)=\phi(\omega)(s)$. Clearly to specify $\phi$ it suffices to specify $\phi_{s}$ for each $s \in[0,1]$ in such a way that $s \rightarrow \phi_{s}(\omega)$ is continuous for each $\omega \in \Omega$. Finally, we denote by $\phi_{*} P$ the measure on $W(Q)$ such that $\phi_{*} P(A)=P\left(\phi^{-1}(A)\right)$ for all $A \subset W(Q)$ such that $\phi^{-1}(A) \in \mathscr{F}$. That is to say $\phi_{*} P$ is the law of $\phi$ under the measure $P$. For the rest of this section we restrict our attention to $Q=M$ and $q_{o}=o$.

For each $s \in[0,1]$ let $\Sigma_{s}: W(M) \rightarrow M$ denote the coordinate functions given by $\Sigma_{s}(\sigma)=\sigma(s)$. (Note under the above convention, $\Sigma$ can be identified with the identity map from $W(M)$ to $W(M)$.) Let $\mathscr{G}_{s}^{\circ}$ be the $\sigma$-algebra on $W(M)$ generated by the coordinate maps $\left\{\Sigma_{u}: u \leq s\right\}$, and $\mathscr{G}^{\circ}$ be the $\sigma$ algebra $\mathscr{G}_{1}^{o}$. We will refer to $\mathscr{G}^{o}$ as the raw $\sigma$-algebra and $\left\{\mathscr{G}_{s}^{o} \mid s \in[0,1]\right\}$ as the raw filtration on $W(M)$. The next theorem supplies the definition and some of the basic properties of Wiener and pinned Wiener measure on $\left(W(M), \mathscr{G}^{\circ}\right)$.

Theorem 2.3. There exist two finite measures $(\nu)$ and $\left(\nu_{e}\right)$ on $\left(W(M), \mathscr{G}^{\circ}\right)$ which are uniquely determined by specifying their finite dimensional distributions as follows. For all $k \in\{1,2,3, \ldots\}$, partitions $0=s_{0}<s_{1}<s_{2}<\cdots<$ $s_{k-1}<s_{k}=1$ of $[0,1]$, and for all bounded measurable functions $f: M^{k} \rightarrow \mathbb{R}$;

$$
\begin{aligned}
& \nu\left(f\left(\Sigma_{s_{1}}, \ldots, \Sigma_{s_{k}}\right)\right) \\
& \quad=\int_{M^{k}} f\left(x_{1}, \ldots, x_{k}\right) \prod_{i=1}^{k} p_{\Delta s_{i}}\left(x_{i-1}, x_{i}\right) d x_{1} \cdots d x_{k},
\end{aligned}
$$

and

$$
\begin{aligned}
& \nu_{e}\left(f\left(\Sigma_{s_{1}}, \ldots, \Sigma_{s_{k}}\right)\right) \\
& \quad=\int_{M^{k-1}} f\left(x_{1}, \ldots, x_{k-1}, e\right) \prod_{i=1}^{k} p_{\Delta s_{i}}\left(x_{i-1}, x_{i}\right) d x_{1} \cdots d x_{k-1},
\end{aligned}
$$


where $x_{0} \equiv o, \Delta s_{i} \equiv s_{i}-s_{i-1}, \quad p_{t}(x, y)$ is the heat kernel, and $x_{k} \equiv e$ in (2.6). (Informally $\nu_{e}(\cdot)=\nu\left(\cdot \mid \Sigma_{1}=e\right) \cdot p_{1}(o, e)$.) Furthermore the two measures $(\nu)$ and $\left(\nu_{e}\right)$ enjoy the following properties:

(1) Both measures $\nu$ and $\nu_{e}$ are concentrated on the set of Hölder continuous paths for any Hölder coefficient $\alpha<1 / 2$.

(2) The two measures $\nu$ and $\nu_{e}$ are equivalent on $\mathscr{G}_{s}^{o}$ for each $s<1$. In fact, $\nu_{e}(F)=\nu\left(p_{1-s}\left(\Sigma_{s}, e\right) F\right)$ for all bounded $\mathscr{G}_{s}^{o}$-measurable functions.

(3) The process $\left\{\Sigma_{s}\right\}_{0 \leq s \leq 1}$ is an $M$-valued Brownian motion with respect to the filtered probability space $\left(W(M),\left\{\mathscr{G}_{s}^{\circ}\right\}, \mathscr{G}^{\circ}, \nu\right)$. Recall that this means for all $f \in\left(C^{\infty}(M)\right)$, the process

$$
M_{s}^{f} \equiv f\left(\Sigma_{s}\right)-f\left(\Sigma_{0}\right)-\frac{1}{2} \int_{0}^{s} \Delta f\left(\Sigma_{s^{\prime}}\right) d s^{\prime}
$$

is a martingale.

(4) The process $\left\{\Sigma_{s}\right\}_{0 \leq s \leq 1}$ is an $M$-valued semimartingale with respect to the filtered probability space $\left(W(M),\left\{\mathscr{G}_{s}^{o}\right\}, \mathscr{G}^{o}, \nu_{e}\right)$. Furthermore for all $f \in C^{\infty}(M)$, the process

$$
\begin{aligned}
N_{s}^{f} \equiv & f\left(\Sigma_{s}\right)-f\left(\Sigma_{0}\right)-\frac{1}{2} \int_{0}^{s} \Delta f\left(\Sigma_{s^{\prime}}\right) d s^{\prime} \\
& -\int_{0}^{s} d f\left\langle\nabla \log p_{1-s^{\prime}}\left(\Sigma_{s^{\prime}}, e\right)\right\rangle d s^{\prime}
\end{aligned}
$$

is a martingale.

Remark 2.4. Notice that the measure $\nu$ is concentrated on $W_{o}(M)$ and the measure $\nu_{e}$ is concentrated on the set of pinned paths in $W_{o}(M)$ which end at $e \in M$.

Proof. Most of the results in this theorem are standard. Item 4 goes under the theory of Doob's " $h$-transformation"; see Doob [12]. To my knowledge the proof that $N_{s}^{f}$ is a martingale for $s \in[0,1]$ including $s=1$ was first given by Bismut in [4]. For a more general treatment of this issue see Yor [56], and the related articles in the same lecture notes. The reader may also wish to consult Jones and Leandre [25].

For the sake of completeness I will sketch the proof of this theorem. There are of course a number of different proofs for each part.

The first step is the proof of existence of $\nu$ and $\nu_{e}$. I will only prove the existence of $\nu_{e}$ since the argument for $\nu$ is similar. Using Kolmogorov's extension theorem (see for example [53]) it is easy to conclude there exists an $M$-valued process $X_{s}$ on some probability space $(\Omega, \mathscr{F}, P)$ such that finite dimensional distributions of $\left\{X_{s}\right\}$ agree with those proposed for $\nu_{e}$ in (2.6). If we can show that the process $\left\{X_{s}\right\}$ has a continuous version (which will still be called $X_{s}$ ), then the law of the process $\left\{X_{s}\right\}$ will be the desired measure $\nu_{e}$. To this end we will apply Kolmogorov's lemma, see for example [44, 46] or [53]. In order to use this lemma it is necessary to estimate $P\left(d\left(X_{s}, X_{t}\right)^{k}\right)$, where $0 \leq s, t \leq 1$, and $k$ is a positive integer. (The following argument is analogous to the proof of Proposition 2.1 in Epperson and Lohrenz [15].) We consider the case where $\frac{1}{4} \leq s \leq t<1$, the other cases can be handled 
similarly. For $s$ and $t$ in this range we have

$$
\begin{aligned}
P\left(d\left(X_{s}, X_{t}\right)^{k}\right) & =\int_{M^{2}} p_{s}(o, x) p_{t-s}(x, y) p_{1-t}(y, e) d(x, y)^{k} d x d y \\
& \leq C \int_{M^{2}}(t-s)^{-d / 2} e^{-c d(x, y)^{2} /(t-s)} p_{1-t}(y, e) d(x, y)^{k} d x d y,
\end{aligned}
$$

where we have used the Gaussian upper bound in (2.1) twice. Now concentrate on estimating the $x$-integral. By compactness of $M$, it is easy to see that there is an $\epsilon>0$ (independent of $m \in M$ ) such that the (geodesic) exponential map (exp) restricted to $T_{m} M$ is a diffeomorphism on $B_{m}(\epsilon)$-the ball in $T_{m} M$ centered at $0_{m} \in T_{m} M$ of radius $\epsilon$. For $v \in T_{m} M$, let $\rho_{m}(v)$ denote the density of the pullback of the Riemannian volume form on $M$ by $\exp _{m}$ with respect to the volume form on $T_{m} M$. Clearly $\rho_{m}(v)$ is a continuous function on the $\epsilon$-neighborhood of the 0 -section of $T M$. Therefore, by reducing $\epsilon$ if necessary we may assume there are constants $0<a<b<\infty$ such that $a \leq \rho_{m}(v) \leq b$ for all $m \in M$ and $v \in B_{m}(\epsilon)$. Using these comments and passing to polar coordinates one finds (for $\delta>0$ ) the estimate

$$
\begin{aligned}
\int_{M} \delta^{-d / 2} \cdot e^{-c d(x, y)^{2} / \delta} d(x, y)^{k} d x \\
\quad \leq \int_{B_{y}(\epsilon)} \delta^{-d / 2} e^{-c d(x, y)^{2} / \delta} d(x, y)^{k} d x+\delta^{-d / 2} O\left(e^{-c \epsilon^{2}}\right) \\
\quad \leq K \int_{0}^{\epsilon} \delta^{-d / 2} e^{-c r^{2} / \delta} r^{k} r^{d-1} d r+\delta^{-d / 2} O\left(e^{-c \epsilon^{2} / \delta}\right) \\
\quad \leq C(k) \delta^{k / 2}
\end{aligned}
$$

where the constant $C(k)$ only depends on k. Combining 2.9 and 2.10 with $\delta=t-s$ yields upon integrating out the $y$-variable (using the symmetry of $p_{s}(x, y)$ and the fact that $\int_{M} p_{s}(x, y) d y=1$ for all $\left.x \in M\right)$

$$
P\left(d\left(X_{s}, X_{t}\right)^{k}\right) \leq C(k)|t-s|^{k / 2} .
$$

By similar arguments this last estimate is easily seen to be valid for all $s$ and $t$ in $[0,1]$. It is now an easy matter to apply Kolmogorov's Lemma to conclude there is a continuous version of $\left\{X_{s}\right\}$ and in fact this version is $\alpha$-Hölder continuous for all $\alpha<1 / 2$. This proves the existence of $\nu_{e}$ and also item 1 in the theorem. The same techniques also work for the measure $\nu$. The unicity of the measures $\nu$ and $\nu_{e}$ satisfying (2.5) and (2.6) respectively follows easily from the fact that "smooth cylinder functions" of the form $F=f\left(\Sigma_{s_{1}}, \ldots, \Sigma_{s_{k}}\right)$ generate the $\sigma$-algebra $\mathscr{G}^{\circ}$.

It is clear that $\nu_{e}(F)=\nu\left(p_{1-s}\left(\Sigma_{s^{\bullet}}, e\right) F\right)$ when $F$ is a smooth cylinder function on $W(M)$ of the form $F=f\left(\Sigma_{s_{1}}, \ldots, \Sigma_{s_{k}}\right)$ with $0 \leq s_{1}, \ldots, s_{k} \leq$ $s<1$. Since such functions generate $\mathscr{G}_{s}^{o}$, it follows that $\nu_{e}=p_{1-s}\left(\Sigma_{s}, \cdot\right) \cdot \nu$ on $\mathscr{G}_{s}^{o}$ for all $s<1$. This proves item 2 .

Item 3 in the theorem is well known from the theory of Markov processes, see [53]. Indeed, using the the Markovian property of $\nu$ one computes for $s>t$, 
$F$ a bounded measurable $\mathscr{G}_{t}^{o}$-measurable function, and $f \in\left(C^{\infty}(M)\right)$ :

$$
\begin{aligned}
\frac{d}{d s} \nu\left(f\left(\Sigma_{s}\right) F\right) & =\frac{d}{d s} \nu\left(\left(e^{(s-t) \Delta / 2} f\right)\left(\Sigma_{t}\right) F\right) \\
& \left.=\frac{1}{2} \nu\left(e^{(s-t) \Delta / 2} \Delta f\right)\left(\Sigma_{t}\right) F\right)=\nu\left(\frac{1}{2} \Delta f\left(\Sigma_{s}\right) F\right) .
\end{aligned}
$$

Integrating this last expression from $t$ to $s$ shows that

$$
\nu\left(\left[M_{t}^{f}-M_{s}^{f}\right] F\right)=\nu\left(\left\{f\left(\Sigma_{t}\right)-f\left(\Sigma_{s}\right)-\int_{s}^{t} \frac{1}{2} \Delta f\left(\Sigma_{s^{\prime}}\right) d s^{\prime}\right\} F\right)=0,
$$

which shows that $M^{f}$ is a martingale.

More generally, the computation done above can be extended to the case that $f$ is a function of $(s, x) \in[0,1) \times M$. One then finds for $s>t$ that

$$
\frac{d}{d s} \nu\left(f\left(s, \Sigma_{s}\right) \cdot F\right)=\nu\left(\left(\frac{\partial}{\partial s}+\frac{1}{2} \Delta\right) f\left(s, \Sigma_{s}\right) \cdot F\right) .
$$

Now let $h(s, x) \equiv p_{1-s}(x, e)$. Then $h$ satisfies $\partial h / \partial s+\frac{1}{2} \Delta h=0$ on $[0,1) \times$ $M$. Hence using (2.12), for $s>t$ one computes

$$
\begin{aligned}
\frac{d}{d s} \nu_{e}\left(f\left(\Sigma_{s}\right) F\right) & =\frac{d}{d s} \nu\left(h\left(s, \Sigma_{s}\right) f\left(\Sigma_{s}\right) F\right)=\nu\left(\left(\frac{\partial}{\partial s}+\frac{1}{2} \Delta\right)\left[h\left(s, \Sigma_{s}\right) f\left(\Sigma_{s}\right)\right] F\right) \\
& =\nu\left(\left[\nabla h\left(s, \Sigma_{s}\right) \cdot \nabla f\left(\Sigma_{s}\right)+h\left(s, \Sigma_{s}\right) \frac{1}{2} \Delta f\left(\Sigma_{s}\right)\right] F\right) \\
& =\nu_{e}\left(\left[\nabla \log h\left(s, \Sigma_{s}\right) \cdot \nabla f\left(\Sigma_{s}\right)+\frac{1}{2} \Delta f\left(\Sigma_{s}\right)\right] F\right) .
\end{aligned}
$$

It now follows by integrating (2.13), as was done for $M^{f}$, that $N_{s}^{f}$ defined in equation (2.8) is a martingale for $s<1$. Because of the estimate (2.2) and item 1 of this theorem, it is clear that the integral in (2.8) is absolutely convergent $\nu_{e}$-a.e. even for $s=1$. Therefore $N_{s}^{f}$ is a local martingale for $s \in[0,1]$. We have already seen that $\nu_{e}\left(d\left(\Sigma_{1-s}, e\right)^{k}\right) \leq C(k)(1-s)^{k / 2}$; see equation $(2.11)$. Using this estimate and the gradient estimate (2.2), one easily shows that $\left|N_{s}^{f}\right|$ is bounded by a fixed function $(g)$ which is independent of $s$ and satisfies $\nu_{e}\left(|g|^{k}\right) \leq C(k, f) \int_{0}^{1}(1-s)^{-k / 2}<\infty$ provided that $k<2$. Therefore, $N_{s}^{f}$ is in fact a uniformly integrable martingale on $[0,1]$.

2.4. Filtration technicalities. For technical reasons when doing stochastic calculus it is convenient to work with filtered probabilities spaces $(\Omega,\{\mathscr{F}\}, \mathscr{F}, P)$ which satisfy the "usual hypothesis." That is $\mathscr{F}$ should be complete with respect to $P, \mathscr{F}_{s}$ should contain all $P$-null sets, and the filtration $\left\{\mathscr{F}_{s}\right\}$ should be right continuous. Since the two measures $\nu$ and $\nu_{e}$ are not equivalent with respect to one another on $\mathscr{G}^{\circ}$, it is not possible to complete $\left(W(M),\left\{\mathscr{G}_{s}^{\circ}\right\}, \mathscr{G}^{\circ}\right)$ in such a way that the usual hypothesis will hold simultaneously for both measures $\nu$ and $\nu_{e}$. However for our purposes, it will suffice to have the usual hypothesis hold with respect to the measure $\bar{\nu} \equiv \nu+\nu_{e}$. We recall the following standard augmentation process. 
Definition 2.5. Let $\left(\Omega,\left\{\mathscr{F}_{s}\right\}, \mathscr{F}, P\right)$ be a filtered measure space. The completion of $\mathscr{F}$ with respect to $P$ is denoted by $\mathscr{F}^{P}$. The measure $P$ extended to $\mathscr{F}^{P}$ will still be denoted by $P$. Let $\mathscr{N}^{P}$ denote the $P$-null sets in $\mathscr{F}^{P}$ and $\mathscr{F}_{s}^{P} \equiv \sigma\left(\mathscr{F}_{s} \cup \mathscr{N}^{P}\right)$, the augmented filtration.

In order to simplify notation we write $\mathscr{N}$ for $\mathscr{N}^{\bar{\nu}}, \mathscr{G}$ for $\left(\mathscr{G}^{\circ}\right)^{\bar{\nu}}$, and $\mathscr{G}_{s}$ for $\left(\mathscr{G}_{s}^{o}\right)^{\bar{\nu}}$. Since $\nu$ and $\nu_{e}$ are absolutely continuous with respect to $\bar{\nu}$, it follows that both $\nu$ and $\nu_{e}$ have natural extensions to $\mathscr{G}$. These extensions will still be denoted by $\nu$ and $\nu_{e}$ respectively.

Proposition 2.6. Using the above notation:

(1) The $\sigma$-algebra $\mathscr{G}_{s}$ may alternatively be described as $\mathscr{G}_{s}=\{A \subset W(M)$ : $\left.\exists B \in \mathscr{G}_{s}^{\circ} \ni A \Delta B \in \mathcal{N}\right\}$, where $A \Delta B \equiv(A \backslash B) \cup(B \backslash A)$.

(2) The measures $\nu, \nu_{e}$, and $\bar{\nu}$ are equivalent on $\mathscr{G}_{s}$ for all $s<1$.

(3) The $\sigma$-algebras $\mathscr{G}_{s}$ for $s<1$ are complete with respect to both $\nu$ and $\nu_{e}$.

(4) The filtration $\left\{\mathscr{G}_{s}\right\}_{0 \leq s \leq 1}$ is right continuous.

Proof. The first three items in the theorem are routine and left to the reader. The last item is well known in Markov theory and I will only briefly sketch the argument.

Let $s<t$ be in $[0,1]$, and assume that $Z$ is a bounded smooth cylinder function of the form $Z=f\left(\Sigma_{s_{1}}, \ldots, \Sigma_{s_{k}}, \Sigma_{s_{k+1}} \ldots, \Sigma_{s_{k+n}}\right)$, where $0=s_{1}<$ $s_{2}<\cdots<s_{k}=s<s_{k+1}<\cdots<s_{k+n}=1$. I claim that $\nu\left(Z \mid \mathscr{G}_{s+}^{o}\right)=\nu\left(Z \mid \mathscr{G}_{s}^{o}\right) \nu-$ a.s. To see this choose $t \in\left(s, s_{k+1}\right)$, and compute $\nu\left(Z \mid \mathscr{G}_{t}{ }^{\circ}\right)$ using the Markov property to get

$$
\nu\left(Z \mid \mathscr{G}_{t}^{o}\right)=g\left(\Sigma_{s_{1}}, \ldots, \Sigma_{s_{k}}, \Sigma_{t} ; t\right),
$$

where

$$
g\left(x_{1}, \ldots, x_{k}, y_{0} ; t\right) \equiv \int_{M^{n}} f\left(x_{1}, \ldots, x_{k}, y_{1}, \ldots, y_{n}\right) \prod_{i=1}^{n}\left(p_{\Delta s_{i}}\left(y_{i-1}, y_{i}\right) d y_{i}\right),
$$

$\Delta s_{i} \equiv s_{i+k}-s_{i+k-1}$ for $i>1$, and $\Delta s_{1} \equiv s_{k+1}-t$. Because the heat kernel is smoothing the function $g\left(x_{1}, \ldots, x_{k}, y_{0} ; t\right)$ is still smooth in all of its variables. Therefore

$$
\nu\left(Z \mid \mathscr{G}_{s+}^{o}\right)=\lim _{t \downarrow s} \nu\left(Z \mid \mathscr{G}_{t}^{o}\right)=g\left(\Sigma_{s_{1}}, \ldots, \Sigma_{s_{k}}, \Sigma_{s} ; s\right)=\nu\left(Z \mid \mathscr{G}_{s}^{o}\right),
$$

for all smooth cylinder functions $Z$. By a monotone class argument one concludes that $\nu\left(Z \mid \mathscr{G}_{s+}^{o}\right)=\nu\left(Z \mid \mathscr{G}_{s}^{o}\right) \quad \nu$-a.s., where $Z$ is any bounded $\mathscr{G}^{\circ}$ measurable function. From this observation and item 1 of this proposition, it follows that $\nu\left(Z \mid \mathscr{G}_{s+}\right)=\nu\left(Z \mid \mathscr{G}_{s}\right) \quad \nu$-a.s. for all bounded $\mathscr{G}$-measurable functions $Z$ and $s<1$. So if $s<1$ and $Z$ is a bounded $\mathscr{G}_{s+}$-measurable function, then $Z=\nu\left(Z \mid \mathscr{G}_{s+}\right)=\nu\left(Z \mid \mathscr{G}_{s}\right)$. Hence $Z$ is equal to a $\mathscr{G}_{s}$-measurable function up to a $\nu$-null set in $\mathscr{G}_{s+}$. By item 2 one concludes that $Z$ is equal to a $\mathscr{G}_{s^{-}}$ measurable function up to a $\bar{\nu}$-null set. Therefore, $Z$ is in fact $\mathscr{G}_{s}$-measurable, because $\left(W(M),\left\{\mathscr{G}_{s}\right\}, \mathscr{G}, \bar{\nu}\right)$ satisfies the usual hypothesis.

2.5. The stochastic development map. The reader who finds this section too abbreviated should consult $\S 8$ of Driver [13]. In this section I will only remark 
here on the minor modifications needed to generalize the discussion in [13] to cover the applications needed in this article.

We will need some more notations. For $s \in[0,1]$ let $B_{s}: W\left(\mathbb{R}^{d}\right) \rightarrow \mathbb{R}^{d}$ denote the coordinate map $B_{s}(\omega) \equiv \omega(s)$, and $\mathscr{F}_{s}{ }^{o}$ be the $\sigma$-algebra on $W\left(\mathbb{R}^{d}\right)$ generated by $B_{t}$ for $t \leq s$. (Again $\mathscr{F}_{s}{ }^{\circ}$ will be called the raw filtration and $\mathscr{F}^{o} \equiv \mathscr{F}_{1}^{0}$ will be called the raw $\sigma$-algebra on $W\left(\mathbb{R}^{d}\right)$.)

Definition 2.7. Relative to the filtered probability space $\left(W(M),\left\{\mathscr{G}_{s}\right\}, \mathscr{G}, \bar{\nu}\right)$, let $\hat{\Psi}: W(M) \rightarrow W\left(\mathbb{R}^{d}\right)$ be a fixed version of $\int \theta\left\langle\delta H_{s}\right\rangle$, where $H_{s}$ is the stochastic horizontal lift of $\left\{\Sigma_{s}\right\}$ starting at $u_{o}$ and $\delta H$ denotes the Stratonovich differential of $H$.

Remarks 2.8. (1) Because the coordinate process $\left\{\Sigma_{s}\right\}$ is a semimartingale relative to both of the measures $\nu_{e}$ and $\nu$ it follows that $\left\{\Sigma_{s}\right\}$ is a semimartingale relative to the measure $\bar{\nu}$ also. This follows easily from Theorem 3, p. 45 of Protter [44] and the definition of an $M$-valued semimartingale.

(2) Because $\nu$ and $\nu_{e}$ are absolutely continuous relative to $\bar{\nu}$, it follows that $\hat{\Psi}$ is a version of $\int \theta\left\langle\delta H_{s}\right\rangle$ where all stochastic integrals and differential equations are taken with respect to either $\nu$ or $\nu_{e}$.

Definition 2.9. The measures $\mu, \mu_{e}$, and $\bar{\mu}$ on $\left(W\left(\mathbb{R}^{d}\right), \mathscr{F} o\right)$ are defined to be $\mu=\widehat{\Psi}_{*} \nu, \mu_{e}=\widehat{\Psi}_{*} \nu_{e}$, and $\bar{\mu}=\widehat{\Psi}_{*} \bar{\nu}=\mu+\mu_{e}$. To simplify notation we write $\mathscr{F}$ for $(\mathscr{F} o)^{\bar{\mu}}, \mathscr{F}_{s}$ for $\left(\mathscr{F}_{s}^{o}\right)^{\bar{\mu}}$, and $\widetilde{\mathscr{N}}$ for $\mathscr{N}^{\bar{\mu}}$.

It is well known that $\mu=\widehat{\Psi}_{*} \nu$ is nothing other than the standard Wiener measure on $W\left(\mathbb{R}^{d}\right)$; see for example Emery [14, Proposition 8.26].

Proposition 2.10. Let $\widehat{\Psi}: W(M) \rightarrow W\left(\mathbb{R}^{d}\right)$ be as above, then:

(1) The measures $\mu, \mu_{e}$, and $\bar{\mu}$ are all equivalent on $\mathscr{F}_{s}$ for each $s<1$.

(2) For each $s<1$ the $\sigma$-algebra $\mathscr{F}_{s}$ is complete with respect to $\mu, \mu_{e}$, and $\bar{\mu}$.

(3) The filtration $\left\{\mathscr{F}_{s}\right\}$ is right continuous.

(4) The map $\widehat{\Psi}: W(M) \rightarrow W\left(\mathbb{R}^{d}\right)$ is $\mathscr{G}_{s} / \mathscr{F}_{s}$-measurable for all $s \in[0,1]$.

(5) The coordinate process $\left\{B_{s}\right\}$ on $W\left(\mathbb{R}^{d}\right) \quad\left(B_{s}(\omega) \equiv \omega(s)\right)$ is a semimartingale relative to the filtered measure space $\left(W(M),\left\{\mathscr{F}_{s}\right\}_{0 \leq s \leq 1}\right.$, $\left.\mathscr{F}, \mu^{*}\right)$, where $\mu^{*}$ is either $\mu, \mu_{e}$, or $\bar{\mu}$.

Proof. Items 1, 2, and 4 are easy and will be left to the reader. The proof of item 3 is the same as the proof of item 4 in Proposition 2.6. The proof of item 5 follows from the "good integrator" definition of a semimartingale (see [44, p. 44]). See Lemma 8.5. of Driver [13] for more details.

Remark 2.11. We will use Girsanov's theorem in Theorem 2.13 to show that martingale part of $B_{s}$ relative to $\left(W(M),\left\{\mathscr{F}_{s}\right\}_{0 \leq s \leq 1}, \mathscr{F}, \mu_{e}\right)$ is again a Brownian motion.

Theorem 2.12. Relative to the filtered measure space $\left(W\left(\mathbb{R}^{d}\right),\left\{\mathscr{F}_{s}\right\}, \mathscr{F}, \bar{\mu}\right)$, let $\Psi: W\left(\mathbb{R}^{d}\right) \rightarrow W(M)$ be a fixed version of $\pi \circ I$, where $I_{s}$ is the solution to the Stratonovich stochastic differential equation $d I_{s}=B\left\langle\delta B_{s}\right\rangle\left(I_{s}\right)$ with initial condition $I_{o}=u_{o}$. The function $\Psi$ is $\mathscr{F}_{s} / \mathscr{G}_{s}$-measurable for all $s \in[0,1]$ and 
the function $\Psi: W\left(\mathbb{R}^{d}\right) \rightarrow W(M)$ is a measure theoretic isomorphism with inverse $\widehat{\Psi}$ in the following sense:

$$
\bar{\nu}\{\Psi \circ \widehat{\Psi} \neq \mathrm{id}\}=0 \quad \text { and } \quad \bar{\mu}\{\widehat{\Psi} \circ \Psi \neq \mathrm{id}\}=0 .
$$

Proof. The proof is only a minor modification of the proof of Theorem 8.3 in [13].

Theorem 2.13. Let $\Psi$ and $\widehat{\Psi}$ be as in Theorem 2.12 and set

$$
\begin{gathered}
b_{s} \equiv \widehat{\Psi}_{s}=B_{s} \circ \widehat{\Psi}, \\
a_{s} \equiv \int_{0}^{s} H_{s^{\prime}}^{-1} \nabla \log p_{1-s^{\prime}}\left(\Sigma_{s^{\prime}}, e\right) d s^{\prime},
\end{gathered}
$$

and

$$
A_{s} \equiv \int_{0}^{s} I_{s^{\prime}}^{-1} \nabla \log p_{1-s^{\prime}}\left(\Psi_{s^{\prime}}, e\right) d s^{\prime} .
$$

Then $\beta_{s} \equiv b_{s}-a_{s}$ and $\bar{\beta}_{s} \equiv B_{s}-A_{s}$ (for $\left.s \in[0,1]\right)$ are $\mathbb{R}^{d}$-valued Brownian motions relative to the filtered probability spaces $\left(W(M),\left\{\mathscr{G}_{s}\right\}_{0 \leq s \leq 1}, \mathscr{G}, \nu_{e}\right)$ and $\left(W\left(\mathbb{R}^{d}\right),\left\{\mathscr{F}_{s}\right\}_{0 \leq s \leq 1}, \mathscr{F}, \mu_{e}\right)$ respectively.

Proof. In order to simplify notation set $z_{s} \equiv p_{1-s}\left(\Sigma_{s}, e\right)$. As has already been pointed out, it is well known that $b_{s} \equiv \widehat{\Psi}_{s}$ is a Brownian motion relative the filtered probability space $\left(W(M),\left\{\mathscr{G}_{s}\right\}_{0 \leq s \leq 1}, \mathscr{G}, \nu\right)$. Since $\nu_{e}=p_{1-s}\left(\Sigma_{s}, e\right)$. $\nu \equiv z_{s} \cdot \nu$ on $\mathscr{G}_{s}$ for all $s<1$, we know by Girsanov's theorem (see [44]) that $\beta_{s} \equiv b_{s}-\int_{0}^{s} z_{s}^{-1} d\left[z_{s}, b_{s}\right]$ is a $\nu_{e}$-martingale for $s<1$. We now need to compute $d z_{s}$. First recall that $\Sigma=\Psi \circ b$ and that $H=I \circ b \nu$-a.s., so that $d H=B\langle\delta b\rangle(H)$. See $\S 8$ of [13] for more details. To simplify notation let $h:[0,1) \times O(M) \rightarrow \mathbb{R}$ be given by $h(s, u)=p_{1-s}(\pi(u), e)$. Notice because $p_{s}(x, e)$ is a solution to the heat equation on $M$ that $\left(\partial / \partial s+\frac{1}{2} \sum_{i=1}^{d} B_{i}^{2}\right) h \equiv$ 0 , where $B_{i} \equiv B\left\langle e_{i}\right\rangle$ with $\left\{e_{i}\right\}_{i=1}^{d}$ the standard basis on $\mathbb{R}^{d}$. Because $z_{s}=$ $h\left(s, H_{s}\right)$ and the above remarks we find

$$
d z_{s}=B\left\langle d b_{s}\right\rangle\left(H_{s}\right) h(s, \cdot)=H_{s}^{-1} \nabla p_{1-s}\left(\Sigma_{s}, e\right) \cdot d b_{s},
$$

where we have used $B\langle a\rangle(u) h(s, \cdot)=u^{-1} \nabla p_{1-s}(\pi(u), e) \cdot a$. (We are writing $a \cdot b$ for the standard dot product on $\mathbb{R}^{d}$.) Thus

$$
z_{s}^{-1} d z_{s} d b_{s}=H_{s}^{-1} \nabla \log p_{1-s}\left(\Sigma_{s}, e\right) d s
$$

and hence, with $a_{s} \equiv \int_{0}^{s} H_{s^{\prime}}^{-1} \nabla p_{1-s^{\prime}}\left(\Sigma_{s^{\prime}}, e\right) d s^{\prime}$, it follows that $\beta_{s} \equiv b_{s}-a_{s}$ is a $\nu_{e}$-martingale at least for $s<1$. Because $\nu$ and $\nu_{e}$ are equivalent on $\mathscr{G}_{s}$ for $s<1$, it follows that $\beta_{s}$ and $b_{s}$ have the same quadratic variations, i.e. $\left[\beta_{s}^{i}, \beta_{s}^{j}\right]=\delta_{i j} s$. Therefore by Levi's theorem (see for example [44]) $\beta_{s}$ is an $\mathbb{R}^{d}$-valued Brownian motion relative $\nu_{e}$. We already know a priori that $b_{s}$ is a $\nu_{e}$-semimartingale, so that the decomposition of $b$ into $\beta+a$ must be valid for $s=1$ also.

Alternatively, by the same techniques as in the proof of $(2.8)$ it is clear that the integrand in the definition of $a_{s}$ is $\nu_{e}$-a.s. integrable for $0 \leq s \leq 1$. In particular $a_{s}$ is a continuous process of bounded variation (relative to $\nu_{e}$ ) and since $\beta_{s}$ is a Brownian motion for $s<1$ it follows that $\beta_{1} \equiv \lim _{s \uparrow 1} \beta_{s}$ exists 
$\nu_{e}$-a.s.. Therefore, by continuity, the set $\left\{b_{s}=\beta_{s}+a_{s} \forall s \in[0,1]\right\}$ has full $\nu_{e}$-measure.

The proof of the decomposition of $B_{s}$ is similar. The key observations is that on $\mathscr{F}_{s}$ for any $s<1$ the measure $\mu_{e}$ is given by $\mu_{e}=p_{1-s}\left(\Psi_{s}, e\right) \cdot \mu$. Using this fact, the desired decomposition is again a straightforward application of Girsanov's theorem.

\section{THE FLOW AND QUASI-INVARIANCE}

Let $\mathbf{H}$ denote the set of absolutely continuous functions $h:[0,1] \rightarrow \mathbb{R}^{d}$ such that $h(0)=0$ and $h^{\prime} \in L^{2}(d s)$. As in [13] one can consider the following "vector field" $\left(X^{h}\right)$ on $W_{o}(M)$ given by $X^{h}(\sigma)(s) \equiv H_{s}(\sigma) h(s)$, where $\sigma \in$ $W_{o}(M)$ and $H_{s}(\sigma)$ denotes the stochastic horizontal lift of $\left.\sigma\right|_{[0, s]}$ to $O(M)$ starting at $u_{o}$. One should note that this vector field is really an equivalence class of vector fields with any particular representative depending on the particular representative chosen for $H_{s}(\cdot)$. We now recall the Theorem 8.5 from Driver [13].

Theorem 3.1. For each $h \in \mathbf{H} \cap C^{1}$ there exists a function $S^{h}: W(M) \rightarrow W(M)$ having the following properties:

(1) $S^{h}$ is $\bar{G}_{s}^{\nu} / \bar{G}_{s}^{\nu}$-measurable for all $s \in[0,1]$.

(2) There are versions of $S^{\text {th }}$ and its horizontal lifts $H\left(S^{\text {th }}\right)$ such that for $\nu$ almost every $\omega \in W(M)$, the functions $f(t, s) \equiv S_{s}^{t h}(\omega), \partial f(t, s) / \partial t$, and $(t, s) \rightarrow H_{s}\left(S_{.}^{\text {th }}(\omega)\right)$ are jointly continuous. Furthermore for $\nu$-a.e. $\omega, S_{s}^{t h}(\omega)$ satisfies

$$
\frac{d}{d t} S_{s}^{t h}(\omega)=H_{s}\left(S_{s}^{t h}(\omega)\right) h(s) \text { with } S_{s}^{0 h}(\omega)=\omega(s)
$$

(3) The functions $\left\{S^{h}\right\}_{h \in \mathbf{H} \cap C^{1}}$ satisfy the group property $S^{\tau h} \circ S^{t h}=S^{(t+\tau) h}$ up to $\nu$-indistinguishability, for all $t, \tau \in \mathbb{R}$.

(4) The measures $\nu^{h} \equiv S_{*}^{h} \nu$ and $\nu$ are equivalent. Furthermore for $s \in$ $[0,1]$, the Radon-Nikodym $Z_{s}^{h}=d \nu^{h} /\left.d \nu\right|_{\mathscr{G}_{s}}$ of $\left.\nu^{h}\right|_{\mathscr{G}_{s}^{\nu}}$ with respect to $\left.\nu\right|_{\mathscr{G}_{s}}$ is

$$
Z_{s}^{h}=\exp \left[-\int_{0}^{s} \alpha^{-h} \cdot O^{-h} d b-\frac{1}{2} \int_{0}^{s}\left|\alpha^{-h}(s)\right|^{2} d s\right],
$$

In (3.2) $\left(\alpha^{h}, O^{h}\right)$ is a $\mathbb{R}^{d} \times O(d)$-valued continuous $\mathscr{G}_{s}^{\nu}$-adapted process and $b$ is the $\nu$-Brownian motion $b \equiv \widehat{\Psi}$. The process $\left(\alpha^{h}, O^{h}\right)$ can be defined by the equation

$$
\widehat{\Psi} \circ S_{s}^{h}=\int_{0}^{s} O_{s^{\prime}}^{h} d b_{s^{\prime}}+\int_{0}^{s} \alpha_{s^{\prime}}^{h} d s^{\prime}
$$

(5) There is a constant $C$ independent of $h \in \mathbf{H} \cap C^{1}$ such that $\nu$-a.s.

$$
\left|\alpha_{s}^{h}\right| \leq C\left\{\left|h^{\prime}(s)\right|+|h(s)|\right\} .
$$

Remark 3.2. The estimate (3.4) is not explicitly stated in Theorem 8.5. of [13]. However, this estimate follows easily from Lemma 6.1. of [13] using $\alpha_{o}=0$, 
the assumption that $\nabla$ is TSS, and the fact that $\alpha^{\text {th }}$ solves equation (6.6) of [13].

Now let $\mathbf{H}_{o}$ denote the set of $h \in \mathbf{H}$ such that $h(1)=0$. In the next proposition we choose a nice version of the flow $S^{h}$ for $h \in \mathbf{H}_{o} \cap C^{1}$.

Proposition 3.3. Keep the same notation as in Theorem 3.1. For $h$ restricted to lie in $\mathbf{H}_{o} \cap C^{1}$ it is possible to choose a versions of $S^{h}$ in Theorem 3.1 such that

(1) $S^{h}$ is $\mathscr{G}_{s} / \mathscr{G}_{s}^{o}$-measurable for all $s \in[0,1]$ (we will see later that $S^{h}$ is in fact $\mathscr{G}_{s} / \mathscr{G}_{s}$-measurable),

(2) $\nu_{e}$-a.s. $d\left(S_{s}^{h}, \Sigma_{s}\right) \leq|h(s)|$ and in particular $\lim _{s \uparrow 1} S_{s}^{h}=e$,

(3) the process $\left(O^{h}, \alpha^{h}\right)$ may be chosen to be $\mathscr{G}_{s}$-adapted for $s \in[0,1)$.

Proof. By Proposition 2.6, for each $s_{o} \in[0,1)$ the filtered probability space $\left(W(M),\left\{\mathscr{G}_{s}\right\}_{s \leq s_{o}}, \mathscr{G}_{s_{o}}, \nu\right)$ satisfies the usual hypothesis. Therefore one can construct a function ${ }^{s_{o}} S^{h}$ which is $\mathscr{G}_{\mathrm{s}} / \mathscr{G}_{s}{ }^{o}$-measurable for all $s \leq s_{o}$ and satisfies items 2-5 in Theorem 3.1 for $s \leq s_{o}$. (This is Theorem 3.1 with $[0,1]$ replaced by $\left[0, s_{o}\right]$.) Now choose a sequence $\left\{s_{n}\right\} \subset[0,1)$ such that $s_{n}$ increases to 1 as $n \rightarrow \infty$. Let ${ }^{n} S^{h}={ }^{s_{n}} S^{h}$, with ${ }^{s_{n}} S^{h}$ described above. For any pair of positive integers $(m, n)$ set

$$
\Omega_{m, n}=\left\{\omega \in W(M):{ }^{n} S_{s}^{h}(\omega)={ }^{m} S_{s}^{h}(\omega) \forall s \in\left[0, s_{n} \wedge s_{m}\right]\right\} .
$$

Because of the uniqueness of the functions $S^{h}$ in Theorem 3.1 (see [13, Corollary 6.3]) it follows that $\nu\left(\Omega_{m, n}\right)=1$. Clearly $\Omega_{m, n}$ is $\mathscr{G}_{s_{n} \vee s_{m}}$-measurable, and hence it also follows that $\nu_{e}\left(\Omega_{m, n}\right)=\nu_{e}(1)$ for all $(m, n)$. Let $\Omega_{o} \equiv$ $\bigcap_{m, n=1}^{\infty} \Omega_{m, n}$. Then $\nu\left(\Omega_{o}\right)=\nu_{e}\left(\Omega_{o}\right) / \nu_{e}(1)=1$ and in particular $\Omega_{o} \in \mathscr{G}_{0}$. For $s<1$ define

$$
S_{s}^{h}(\omega)= \begin{cases}\lim _{n \rightarrow \infty}{ }^{n} S_{s}^{h}(\omega) & \text { for } \omega \in \Omega_{o}, \\ \omega_{o} & \text { for } \omega \notin \Omega_{o},\end{cases}
$$

where $\omega_{o}$ is a fixed path in $W(M)$ for which $\omega_{o}(0)=o$ and $\omega_{o}(1)=e$. It is easy to see that $S^{h}$ in (3.5) is $\mathscr{G}_{s} / \mathscr{G}_{s}^{o}$-measurable for all $s<1$.

Because of equation (3.1), the fact that $H_{s}\left(S_{\cdot}^{t h}\right) \in O(M)$, and the definition of the Riemannian distance $d$ on $M$ it easily follows for each $s \in[0,1]$ that

$$
\left|d\left(S_{s}^{h}, \Sigma_{s}\right)\right| \leq|h(s)| \text { holds } \nu \text {-a .s. }
$$

Now for $s<1$ the set $\left\{\left|d\left(S_{s}^{h}, \Sigma_{s}\right)\right| \leq|h(s)|\right\}$ is $\mathscr{G}_{s}$-measurable. Since $\nu$ and $\nu_{e}$ are equivalent on $\mathscr{G}_{s}$, it follows that (3.6) holds $\nu_{e}$-a.e. also. Because both sides of the inequality in (3.6) are continuous it follows that the set

$$
\left\{\left|d\left(S_{s}^{h}, \Sigma_{s}\right)\right| \leq|h(s)| \forall s<1\right\}
$$

has full measure with respect to both $\nu_{e}$ and $\nu$. Because of the assumption that $h \in \mathbf{H}_{o} \cap C^{1}(h(1)=0)$ and the above comments it follows that the set

$$
\Omega_{1} \equiv\left\{\omega: \lim _{s \rightarrow 1} d\left(S_{s}^{h}(\omega), \omega(s)\right)=0\right\}
$$

has full measure with respect to both $\nu$ and $\nu_{e}$. So on $\Omega_{1}$ we may extend the definition of $S^{h}$ (to $s=1$ ) by setting $S_{1}^{h} \equiv \Sigma_{1}$ on $\Omega_{1}$. For $\omega \notin \Omega_{1}$ redefine 
$S_{s}^{h}(\omega)=\omega_{o}(s)$ where $\omega_{o}$ is a fixed path in $W(M)$ such that $\omega_{o}(0)=o$ and $\omega_{o}(1)=e$. It is easily seen that this function $S^{h}$ has all of the properties asserted in the theorem. (Notice that $\Sigma_{1}=e \nu_{e}$-a.s.)

By similar reasoning, one may show that the process $\left(O_{s}^{h}, \alpha_{s}^{h}\right)$ can be chosen to be $\mathscr{G}_{s}$-adapted for all $s<1$. The key point is that $\left(O^{h}, \alpha^{h}\right)$ are unique up to $\nu$-indistinguishability (see Theorem 6.1 in [13]) and so the same arguments as above still apply.

We now come to the main theorem of this paper.

Theorem 3.4. Let $h \in \mathbf{H}_{o} \cap C^{1}$ and $S^{h}$ be as above. For notational simplicity let $\nu_{e}^{h} \equiv S_{*}^{h} \nu_{e}$ on $\mathscr{G}^{\circ}$. Then $\nu_{e}$ and $\nu_{e}^{h}$ are equivalent on $\mathscr{G}^{\circ}$. More precisely $\nu_{e}^{h}=Z^{h} \cdot \nu_{e}$, where

$$
Z^{h}=\exp \left[-\int_{0}^{1} \alpha^{-h} \cdot O^{-h} d b-\frac{1}{2} \int_{0}^{1}\left|\alpha^{-h}(s)\right|^{2} d s\right]
$$

which is the same expression as in (3.2) with $s=1$. However, one should now interpret the stochastic integrals relative to the filtered probability space $\left(W(M),\left\{\mathscr{G}_{s}\right\}, \mathscr{G}, \nu_{e}\right)$ or even better $\left(W(M),\left\{\mathscr{G}_{s}\right\}, \mathscr{G}, \bar{\nu}\right)$. Furthermore, the process $S^{h}$ is $\mathscr{G}_{s} / \mathscr{G}_{s}$-measurable for all $s \in[0,1]$ and $S^{\text {th }} \circ S^{\tau h}=S^{(t+\tau) h} \bar{\nu}$-a.e. for all $t, \tau \in \mathbb{R}$.

Proof. Recall relative to $\nu_{e}$, the process $b$ is no longer a Brownian motion but has the decomposition $b=\beta+a$ given in Theorem 2.13. Now by using item 2 in Theorem 2.3 one easily shows $\nu_{e}^{h}=z_{s} \cdot \nu_{e}$ on $\mathscr{G}_{s}^{o}$ for $s<1$, where

$$
\begin{gathered}
z_{s} \equiv \frac{p_{1-s}\left(S_{s}^{-h}, e\right)}{p_{1-s}\left(\Sigma_{s}, e\right)} Z_{s}^{h} \equiv w_{s} \cdot Z_{s}^{h} \equiv w_{s} \cdot\left(x_{s} \cdot y_{s}\right), \\
w_{s} \equiv \frac{p_{1-s}\left(S_{s}^{-h}, e\right)}{p_{1-s}\left(\Sigma_{s}, e\right)}, \\
x_{s} \equiv \exp \left[-\int_{0}^{s} \alpha^{-h} \cdot O^{-h} d \beta-\frac{1}{2} \int_{0}^{s}\left|\alpha^{-h}(s)\right|^{2} d s\right],
\end{gathered}
$$

and

$$
\begin{aligned}
y_{s} & \equiv \exp \left[-\int_{0}^{s} \alpha^{-h} \cdot O^{-h} d a\right] \\
& =\exp \left[-\int_{0}^{s} \alpha^{-h}\left(s^{\prime}\right) \cdot O^{-h}\left(s^{\prime}\right) H_{s^{\prime}}^{-1} \nabla \log p_{1-s^{\prime}}\left(\Sigma_{s^{\prime}}, e\right) d s^{\prime}\right] .
\end{aligned}
$$

In order to show $\nu_{e}^{h}=Z^{h} \cdot \nu_{e}$, it suffices to show that $z_{s}$ converges to $Z^{h}=$ $Z_{1}^{h}$ in $L^{1}\left(\nu_{e}\right)$ as $s \rightarrow 1$. For then it would follow that $\nu_{e}^{h}(F)=\nu_{e}\left(Z_{1}^{h} F\right)$ where $F$ is any $\mathscr{G}_{s}{ }^{O}$-bounded measurable function and $s \in[0,1)$. By a monotone class argument one can then easily conclude that $\nu_{e}^{h}(F)=\nu_{e}\left(Z_{1}^{h} F\right)$ for all bounded $\mathscr{G}_{1}^{o}=\sigma\left(\bigcup_{s<1} \mathscr{G}_{s}^{o}\right)$-measurable functions. The assertion that $Z_{1}^{h}=L^{1}-\lim _{s \uparrow 1} z_{s}$ is an easy consequence Hölder's inequality and Lemmas 3.5-3.7 below.

We now know on $\mathscr{G}^{\circ}$ that $S_{*}^{h} \nu=Z^{h} \cdot \nu$ and $S_{*}^{h} \nu_{e}=Z^{h} \cdot \nu_{e}$, so it immediately follows that $S_{*}^{h} \bar{\nu}=Z^{h} \cdot \bar{\nu}$ on $\mathscr{G}^{\circ}$ also. It is a trivial exercise to 
show that $\left(S^{h}\right)^{-1}\left(\mathscr{N}^{\bar{\nu}}\right) \subset \mathscr{N}^{\bar{\nu}}$ which, along with the fact that $S^{h}$ is $\mathscr{G}_{s} / \mathscr{G}_{s}^{o}$ measurable for all $s$, implies that $S^{h}$ is $\mathscr{G}_{s} / \mathscr{G}_{s}$-measurable for all $s$. Therefore, given $s \in[0,1)$, the set $A_{s} \equiv\left\{S_{s}^{t h} \circ S^{\tau h} \neq S_{s}^{(t+\tau) h}\right\}$ is $\mathscr{G}_{s}$-measurable. Because of Theorem 3.1, item 3 and Proposition 2.6, item 2, it follows that $\bar{\nu}\left(A_{s}\right)=0$ for all $s \in[0,1)$. Finally because of the continuity of $S_{s}^{h}$ it follows that $\bar{\nu}\left(\left\{S^{t h} \circ S^{\tau h} \neq S^{(t+\tau) h}\right\}\right)=\bar{\nu}\left(\bigcap_{s \in[0,1]} A_{s}\right)=0$. This proves the remaining assertions of the theorem.

Lemma 3.5. $\nu_{e}$-a.s. the process $w_{s}$ in equation (3.11) is bounded and $\lim _{s \uparrow 1} w_{s}=$ 1.

Lemma 3.6. The process $x_{s}$ in equation (3.12) converges to $x_{1}$ in $L^{p}\left(\nu_{e}\right)$ for all $p<\infty$.

Lemma 3.7. The process $y_{s}$ in equation (3.13) converges to $y_{1}$ in $L^{p}\left(\nu_{e}\right)$ for all $p<\infty$.

Proof of Lemma 3.5. By the estimate (2.3) and Proposition 3.3 there exists a constant $C$ such that $\nu_{e}$-a.s.

$$
\left|w_{s}\right|=\left|\frac{p_{1-s}\left(S_{s}^{-h}, e\right)}{p_{1-s}\left(\Sigma_{s}, e\right)}\right| \leq \exp \frac{C d\left(S_{s}^{-h}, \Sigma_{s}\right)}{1-s} \leq \exp \frac{C|h(s)|}{1-s} .
$$

Since by assumption $h(1)=0$ and $h$ is $C^{1}$, it follows that $|h(s)| /(1-s)$ remains bounded for all $s \in[0,1)$, and hence so does $\left|w_{s}\right|$.

From the asymptotic expansion (2.4) one learns that

$$
w_{s}=\exp \left(\frac{-1}{2(1-s)}\left[d\left(S_{s}^{-h}, e\right)^{2}-d\left(\Sigma_{s}, e\right)^{2}\right]\right)\{1+O(1-s)\}
$$

for $s$ sufficiently close to 1 . Now by repeated use of the triangle inequality and Proposition 3.3 one has

$$
\begin{aligned}
\left|d\left(S_{s}^{-h}, e\right)^{2}-d\left(\Sigma_{s}, e\right)^{2}\right| & \leq\left|d\left(S_{s}^{-h}, e\right)-d\left(\Sigma_{s}, e\right)\right| \cdot\left|d\left(S_{s}^{-h}, e\right)+d\left(\Sigma_{s}, e\right)\right| \\
& \leq d\left(S_{s}^{-h}, \Sigma_{s}\right) \cdot\left|d\left(S_{s}^{-h}, e\right)+d\left(\Sigma_{s}, e\right)\right| \\
& \leq|h(s)| \cdot\left\{|h(s)|+2 d\left(\Sigma_{s}, e\right)\right\} .
\end{aligned}
$$

Since $|h(s)| /(1-s)$ remains bounded and because $\nu_{e}$-a.s. $\Sigma_{s} \rightarrow e$ as $s \uparrow 1$, it follows from (3.15) and (3.16) that $\nu_{e}$-a.s. $\lim _{s \uparrow 1} w_{s}=1$.

Proof of Lemma 3.6. Let $\beta=b-a$ be the $\nu_{e}$-Brownian motion defined in Theorem 2.13 and define the $\nu_{e}$-martingale $M_{s}$ by $M_{s} \equiv-\int_{0}^{s} \alpha^{-h} \cdot O^{-h} d \beta$. Then one easily shows that $x_{s}=\exp \left\{M_{s}-\frac{1}{2}[M, M]_{s}\right\}$. Because of equation (3.4), there is a constant $C=C(h)$ such that $[M, M]_{1} \leq C \nu_{e}$-a.s. It is now an easy application of Novikov's criterion to show that $x_{s}$ is a martingale and $\nu_{e}\left(x_{s}^{r}\right) \leq e^{C\left(r^{2}-r\right) / 2}$ for all $r \in \mathbb{R}$; see Remark 8.1. of [13] for more details. By Doob's inequality, for $p>1, x^{*} \equiv \sup _{s \in[0,1]}\left|x_{s}\right|$ satisfies $\nu_{e}\left(x^{* p}\right) \leq$ $(p /(p-1))^{p} e^{C\left(r^{2}-r\right) / 2}$. The lemma is now easily proved using the dominated convergence theorem.

In order to prove Lemma 3.7 we will need the following: 
Lemma 3.8. There is an $\epsilon>0$ such that

$$
\nu_{e}\left(e^{\epsilon\left[\int_{0}^{1} \frac{d\left(\Sigma_{s}, e\right)}{1-s} d s\right]^{2}}\right)<\infty .
$$

Proof. Since $M$ is compact it follows that $\int_{0}^{1 / 2} d\left(\Sigma_{s}, e\right) /(1-s) d s \leq K \log 2$ $<\infty$ where $K=\sup _{m \in M} d(m, e)$. Therefore it suffices to show that there is an $\epsilon>0$ such that

$$
\nu_{e}\left(e^{\epsilon\left[\int_{1 / 2}^{1} \frac{d\left(\mathcal{E}_{s}, e\right)}{1-s} d s\right]^{2}}\right)<\infty .
$$

Now let $\kappa: W(M) \rightarrow W(M)$ denote the map defined by $\kappa(\omega)(s) \equiv \omega(1-s)$ where $s \in[0,1]$. Then if $\nu_{e, o}$ is defined in the same way as $\nu_{e}$ except with $o$ and $e$ interchanged it follows trivially from (2.6) that $\nu_{e}=\kappa_{*} \nu_{e, o}$. Therefore (3.18) may be rewritten as

$$
\kappa_{*} \nu_{e, o}\left(e^{\epsilon\left[\int_{1 / 2}^{1} \frac{d\left(\mathcal{E}_{s}, e\right)}{1-s} d s\right]^{2}}\right)=\nu_{e, o}\left(e^{\epsilon\left[\int_{0}^{1 / 2} \frac{d\left(\mathcal{E}_{s}, e\right)}{s} d s\right]^{2}}\right) .
$$

We may now write the right member of (3.19) as

$$
\hat{\nu}\left(p_{1 / 2}\left(\Sigma_{1 / 2}, o\right) \cdot e^{\epsilon\left[\int_{0}^{1 / 2} \frac{d\left(\Sigma_{s}, e\right)}{s} d s\right]^{2}}\right),
$$

where $\hat{\nu}$ is the Wiener measure on $\left(W(M), \mathscr{G}^{\circ}\right)$ which is concentrated on the paths $\left(W_{e}(M)\right)$ starting at $e \in M$. Since $M$ is compact $p_{1 / 2}(\cdot, o)$ is bounded, and so it now suffices to show that

$$
\exists \epsilon>0 \ni \hat{\nu}\left(e^{\epsilon\left[\int_{0}^{1 / 2} \frac{d\left(\Sigma_{s}, e\right)}{s} d s\right]^{2}}\right)<\infty .
$$

With the aid of the Whitney embedding theorem, one may choose $C^{\infty}$-functions $\left\{D_{i}\right\}_{i=1}^{N}$ such that $D_{i}(e)=0$ and $d(x, e) \leq \sum_{i=1}^{N}\left|D_{i}(x)\right|$ for all $\mathrm{x}$ in $M$. (Indeed, let $M$ be embedded in $\mathbb{R}^{N}$ and set $D_{i}(x)=C\left[y_{i}(x)-y_{i}(0)\right]$ where $C$ is a suitably large constant and $y_{i}$ is the $i$ th standard coordinate function on $\mathbb{R}^{N}$.) Using these remarks, in order to prove (3.21) it suffices to show for each $C^{\infty}$-function $D: M \rightarrow \mathbb{R}$ satisfying $D(e)=0$ that

$$
\exists \epsilon>0 \ni \hat{\nu}\left(e^{\epsilon\left[\int_{0}^{1 / 2} \frac{\mid D\left(\Sigma_{s}\right)}{s} d s\right]^{2}}\right)<\infty .
$$

Now fix such a function $D$, then using the stochastic development in $\S 2.5$ one finds that

$$
D\left(\Sigma_{s}\right)=\int_{0}^{s} H_{s}^{-1} \nabla D\left(\Sigma_{s}\right) \cdot d b_{s}+\int_{0}^{s} \frac{1}{2} \Delta D\left(\Sigma_{s}\right) d s,
$$

where $b_{s}$ is an $\mathbb{R}^{d}$-Brownian motion and $H_{s}$ is a continuous $O(M)$ valued process. Therefore $\left|D\left(\Sigma_{s}\right)\right| \leq K s+\left|\int_{0}^{s} H_{s}^{-1} \nabla D\left(\Sigma_{s}\right) \cdot d b_{s}\right|$, where $K$ is now a bound on $\frac{1}{2} \Delta D$. Set $N_{s} \equiv \int_{0}^{s} H_{s}^{-1} \nabla D\left(\Sigma_{s}\right) \cdot d b_{s}$, so that $N_{s}$ is a continuous martingale. Notice that the quadratic variation satisfies $[N, N]_{s} \leq C s$, where $C$ is a bound for $|\nabla D|$. Putting these comments together we now see it suffices 
to show that

$$
\exists \epsilon>0 \ni E\left(e^{\epsilon\left[\int_{0}^{1 / 2} \frac{\left|N_{s}\right|}{s} d s\right]^{2}}\right)<\infty,
$$

where $N$ is a continuous martingale such that $N_{0}=0$ and $[N, N]_{s} \leq C s$ for some constant $C$.

To verify (3.24) we use the same technique employed to prove Lemma 9.3. in [13]. By the Dambis-Dubins-Schwarz Theorem (see [46, Theorem 1.7, p. 171] or [48, Chapter IV, §34]) there is a continuous martingale $\tilde{N}$ and a Brownian motion $\tilde{B}$ on some probability space $((\Omega, \tilde{F}, \tilde{P}))$ such that $N$ and $\tilde{N}$ have the same law, and $\tilde{N}=\tilde{B}_{[\tilde{N}, \tilde{N}]_{s}}$. Given an $\alpha \in(0,1 / 2)$ set $|f|_{\alpha}=\sup _{s, s^{\prime} \in[0, C]}\left|f(s)-f\left(s^{\prime}\right)\right| /\left|s-s^{\prime}\right|^{\alpha}$, where $f$ is any real valued continuous function on $[0, C]$ such that $f(0)=0$. Then

$$
\left|\tilde{N}_{s}\right|=\left|\tilde{B}\left([\tilde{M}, \tilde{M}]_{s}\right)\right| \leq|\tilde{B}|_{\alpha} \cdot[\tilde{M}, \tilde{M}]_{s}^{\alpha} \leq|\tilde{B}|_{\alpha} \cdot C^{\alpha} s^{\alpha} .
$$

It now follows by elementary calculus that $\int_{0}^{1 / 2}\left|\tilde{N}_{s}\right| / s d s \leq \alpha^{-1} C^{\alpha}|\tilde{B}|_{\alpha}$. Since the expectation in (3.24) satisfies

$$
E\left(e^{\epsilon\left[\int_{0}^{1 / 2} \frac{\left|N_{s}\right|}{s} d s\right]^{2}}\right)=\tilde{E}\left(e^{\epsilon\left[\int_{0}^{1 / 2} \frac{\left|\tilde{N}_{s}\right|}{s} d s\right]^{2}}\right) \leq \tilde{E}\left(e^{\epsilon \alpha^{-2} C^{2 \alpha}|\tilde{B}|_{\alpha}^{2}}\right)
$$

it suffices to show that there is an $\epsilon>0$ such that $\tilde{E} e^{\epsilon|\tilde{B}|_{\alpha}^{2}}<\infty$. But this follows from Fernique's theorem (see [29, pp. 159-160] or [24, p. 402]), since the Hölder seminorms $|\cdot|_{\alpha}$ for $\alpha<1 / 2$ are measurable seminorms for the standard Wiener measure.

Proof of Lemma 3.7. First notice that the integrand in the right-hand member of (3.13) can be estimated (using the estimates (3.4), (2.2), and the assumption that $\left.h \in \mathbf{H}_{o} \cap C^{1}\right)$ by

(3.25) $\left|\alpha^{-h}(s)\right| \cdot\left|\nabla \log p_{1-s}\left(\Sigma_{s}, e\right)\right| \leq K\left[\left|d\left(\Sigma_{s}, e\right)\right| /(1-s)+(1-s)^{-1 / 2}\right]$,

where $K$ is a suitably large constant. Because of item 1 in Theorem 2.3 we know $\nu_{e}$-a.s. that $\left|d\left(\Sigma_{s}, e\right)\right| \leq C(1-s)^{1 / 4}$ for some random constant $C$. Therefore the right member of $(3.25)$ is in $L^{1}([0,1], d s) \nu_{e}$-a.s. This shows $\nu_{e}$-a.s. that $y_{1}$ exists and $\lim _{s \uparrow 1} y_{s}=y_{1}$.

From the definition of $y_{s}$ in (3.13) and the bounds in (3.25) it follows that

$$
\left|y_{s}\right| \leq \exp K\left(\int_{0}^{1}\left|\frac{d\left(\Sigma_{u}, e\right)}{1-u}\right| d u+2\right) .
$$

By Lemma 3.8, one easily shows that all powers of the dominating function on the right side of (3.26) are integrable. Therefore, the lemma is now proved with the aid of the dominated convergence theorem.

3.1. Integration by parts. We conclude this paper with an integration by parts formula which is completely analogous to Theorem 9.1 in Driver [13]. In fact, it is possible to give essentially the same proof but we will instead prove the result using Theorem 9.1 in [13]. Before stating the integration by parts formula we will need some more notation. 
Definition 3.9. For $H \in O(M)$ and $a \in \mathbb{R}^{d}$, define the Ricci tensor by

$$
\operatorname{Ric}_{H}\langle a\rangle \equiv \sum_{i=1}^{d} \Omega_{H}\left\langle a, e_{i}\right\rangle e_{i} \in \mathbb{R}^{d} .
$$

Definition 3.10. For $H \in O(M)$ and $a \in \mathbb{R}^{d}$, set

$$
\widehat{\Theta}_{H}\langle a\rangle \equiv \sum_{i=1}^{d} \boldsymbol{\Theta}_{H}^{\prime}\left\langle e_{i}, a, e_{i}\right\rangle,
$$

where $\left\{e_{i}\right\}_{i=1}^{d}$ is an orthonormal basis for $\mathbb{R}^{d}$, and

$$
\Theta_{H}^{\prime}\langle a, b, c\rangle \equiv B\langle a\rangle(H)\left(u \rightarrow \boldsymbol{\Theta}_{u}\langle b, c\rangle\right) .
$$

Let $\mathscr{K}_{s}^{o}$ be the raw filtration on $W(O(M))$, i.e., the filtration on $W(O(M))$ generated by the coordinate functions $\left\{U_{t}\right\}_{t \in[0, s]}$ where $U_{t}(u)=u(t)$ for all $u \in$ $W(O(M))$. As above, let $H: W(M) \rightarrow W(O(M))$ be a fixed version (relative to the filtered measure space $\left.\left(W(M),\left\{\mathscr{G}_{s}\right\}, \mathscr{G}, \bar{\nu}\right)\right)$ of the horizontal lift of the process $\left\{\Sigma_{s}\right\}$ such that $H_{0}=u_{o}$. It follows automatically that $H$ is $\mathscr{G}_{s} / \mathscr{K}_{s}{ }^{{ }_{-}}$ measurable for all $s \in[0,1]$ and that $H$ is a version of the horizontal lift of $\left\{\Sigma_{s}\right\}$ relative to both measures $\nu_{e}$ and $\nu$.

Definition 3.11 ( $h$-derivative). Let $f: W(M) \rightarrow \mathbb{R}$ be a $C^{2}$-cylinder function, that is $f(\Sigma)=F\left(\Sigma_{s_{1}}, \ldots, \Sigma_{s_{k}}\right)$ for some $0 \leq s_{1} \leq s_{2} \leq \cdots \leq s_{k} \leq 1$ and some $C^{2}$-function $F: M^{k} \rightarrow \mathbb{R}$. (Note: Because $\nu_{e}$-a.s. $\Sigma_{1}=e$, we can and always do assume that $s_{k}<1$.) Then the h-derivative of $f$ is the function $\partial_{h} f: W(M) \rightarrow \mathbb{R}$ given by

$$
\partial_{h} f(\Sigma)=\sum_{i=1}^{k} f_{i}(\Sigma)\left\langle H_{s_{i}}(\Sigma) h\left(s_{i}\right)\right\rangle
$$

where for $v \in T M, f_{i}(\Sigma)\langle v\rangle \equiv v\left(F\left(\Sigma_{s_{1}}, \ldots, \Sigma_{s_{i-1}}, \cdot, \Sigma_{s_{i+1}}, \ldots, \Sigma_{s_{k}}\right)\right.$.

Remark 3.12. As noted in [13], $\partial_{h} f$ is only defined up to $\nu$-equivalence and hence $\nu_{e}$-equivalence. It is also shown in [13] that $\partial_{h} f$ can be defined by $\partial_{h} f=\left.(d / d t)\right|_{0} f \circ S^{t h}$, where the derivative is taken in $\nu$-probability.

Theorem 3.13. Let $h \in \mathbf{H}_{o} \cap C^{1}, b \equiv \widehat{\Psi} \circ \Sigma$, and $H$ be the horizontal lift as above. Then with respect to the $L^{2}\left(\nu_{e}\right)$-inner product the adjoint $\left(\partial_{h}^{*}\right)$ of $\partial_{h}$ contains the set of $C^{2}$-cylinder functions (hence is densely defined). Furthermore if $f$ is a $C^{2}$-cylinder function, then

$$
\partial_{h}^{*} f=-\partial_{h} f+z(h) f,
$$

where $z(h) \equiv \int_{0}^{1}\left[\frac{1}{2} \operatorname{Ric}_{H}\langle h\rangle+\frac{1}{2} \widehat{\Theta}_{H}\langle h\rangle+h^{\prime}\right] \cdot d b$.

Proof. For each $C^{2}$-cylinder function $(f)$, let $\partial_{h}^{*} f$ be given by equation (3.28). It suffices to show that for all $C^{2}$-cylinder functions that

$$
\nu_{e}\left(\partial_{h} f \cdot g\right)=\nu_{e}\left(f \cdot \partial_{h}^{*} g\right) .
$$


According to Theorem 9.1 of Driver [13] the above displayed equation is valid if $\nu_{e}$ is replaced by $\nu$. Therefore if $f$ and $g$ are two $C^{2}$-cylinder functions and $s$ is sufficiently close to 1 we have

$$
\begin{aligned}
\nu_{e}\left(\partial_{h} f \cdot g\right) & =\nu\left(\partial_{h} f \cdot\left(g p_{1-s}\left(\Sigma_{s}, e\right)\right)\right) \\
& =\nu\left(f\left[-\partial_{h}+z(h)\right]\left(g p_{1-s}\left(\Sigma_{s}, e\right)\right)\right) \\
& =\nu\left(f \partial_{h}^{*} g \cdot p_{1-s}\left(\Sigma_{s}, e\right)\right)+\nu_{e}\left(f g \cdot \partial_{h} \ln \left(p_{1-s}\left(\Sigma_{s}, e\right)\right)\right) .
\end{aligned}
$$

So in order to finish the proof it suffices to show that $J \equiv \lim _{s \uparrow 1} \nu\left(f \partial_{h}^{*} g\right.$. $\left.p_{1-s}\left(\Sigma_{s}, e\right)\right)=\nu_{e}\left(f \partial_{h}^{*} g\right)$, and $L \equiv \lim _{s \uparrow 1} \nu_{e}\left(f g \cdot \partial_{h} \ln \left(p_{1-s}\left(\Sigma_{s}, e\right)\right)\right)=0$. We start with $J$.

First notice that $\partial_{h} g$ is still $\mathscr{G}_{s}^{o}$-measurable for $s$ sufficiently close to 1 , and hence $\nu\left(f \partial_{h} g \cdot p_{1-s}\left(\Sigma_{s}, e\right)\right)=\nu_{e}\left(f \partial_{h} g\right)$ for $s$ close to 1 . So to show $J=\nu_{e}\left(f \partial_{h}^{*} g\right)$ it suffices to show $\lim _{s \uparrow 1} \nu\left(f g z(h) p_{1-s}\left(\Sigma_{s}, e\right)\right)=\nu_{e}(f g z(h))$. To this end set

$$
z_{s} \equiv \int_{0}^{s}\left[\frac{1}{2} \operatorname{Ric}_{H}\langle h\rangle+\frac{1}{2} \widehat{\boldsymbol{\Theta}}_{H}\langle h\rangle+h^{\prime}\right] \cdot d b,
$$

where $b=\widehat{\Psi}$ as in Theorem 2.13. Now $b$ is a $\nu$-Brownian motion so that $z_{s}$ is a $\nu$-martingale. Since $f g p_{1-s}\left(\Sigma_{s}, e\right)$ is $\mathscr{G}_{s}^{o}$-measurable for $s$ close to 1 , it follows for $s$ near 1 that

$$
\nu\left(f g z(h) p_{1-s}\left(\Sigma_{s}, e\right)\right)=\nu\left(f g z_{s} p_{1-s}\left(\Sigma_{s}, e\right)\right)=\nu_{e}\left(f g z_{s}\right) .
$$

From this last equation and the fact that $f$ and $g$ are bounded, it now suffices to show that $z_{s} \rightarrow z_{1}=z(h)$ in $L^{1}\left(\nu_{e}\right)$ as $s \uparrow 1$.

To verify that $z_{s} \rightarrow z_{1}$, let $b_{s}=\beta_{s}+a_{s}$ be the decomposition in Theorem 2.13 and decompose $z_{s}=x_{s}+y_{s}$ where

$$
x_{s} \equiv \int_{0}^{s}\left[\frac{1}{2} \operatorname{Ric}_{H}\langle h\rangle+\frac{1}{2} \widehat{\Theta}_{H}\langle h\rangle+h^{\prime}\right] \cdot d \beta
$$

and

$$
y_{s} \equiv \int_{0}^{s}\left[\frac{1}{2} \operatorname{Ric}_{H}\langle h\rangle+\frac{1}{2} \widehat{\Theta}_{H}\langle h\rangle+h^{\prime}\right] \cdot d a .
$$

Since $\beta$ is a $\nu_{e}$-Brownian motion, it is clear that $x_{s}$ is an $L^{2}$-martingale and in particular $x_{s} \rightarrow x_{1}$ in $L^{1}$ as $s \uparrow 1$. We now must show that $y_{s}$ converges to $y_{1}$ in $L^{1}$ as $s \uparrow 1$. To simplify notation, set $R=\left[\frac{1}{2} \operatorname{Ric}_{H}\langle h\rangle+\frac{1}{2} \widehat{\boldsymbol{\theta}}_{H}\langle h\rangle+h^{\prime}\right]$ and notice that $R$ is uniformly bounded since $h \in \mathbf{H}_{o} \cap C^{1}$, and $M$ is compact. With this notation and the definition of $a, y_{s}$ can be written as

$$
y_{s}=\int_{0}^{s} R_{s^{\prime}} H_{s^{\prime}}^{-1} \nabla \log p_{1-s^{\prime}}\left(\Sigma_{s^{\prime}}, e\right) d s^{\prime} .
$$

Using this last equation and the estimate (2.2) one easily shows (with $K=$ $K\left(h^{\prime}\right)>0$ sufficiently large) that:

$$
\left|y_{1}-y_{s}\right| \leq K \int_{s}^{1}\left[\frac{d\left(\Sigma_{s^{\prime}}, e\right)}{\left(1-s^{\prime}\right)}+\sqrt{1-s^{\prime}}\right] d s^{\prime} .
$$

By (2.11) it follows that $\nu_{e}\left(d\left(\Sigma_{s^{\prime}}, e\right)\right) \leq C(1) \sqrt{1-s^{\prime}}$, which combined with (3.30) easily shows that $\nu_{e}\left(\left|y_{1}-y_{s}\right|\right) \leq 2 K(C(1)+1) \sqrt{1-s}$. Hence, $y_{s}$ con- 
verges in $L^{1}\left(\nu_{e}\right)$ to $y_{1}$ as desired. Therefore, $z_{s}=x_{s}+y_{s}$ converges to $z_{1}$ in $L^{1}\left(\nu_{e}\right)$ as claimed and we have shown that $J=\nu_{e}\left(f \partial_{h}^{*} g\right)$.

Now the " $L$ " term is easily shown to be zero as follows. First notice that $\left|\partial_{h} \ln \left(p_{1-s}\left(\Sigma_{s}, e\right)\right)\right| \leq\left|\nabla \ln \left(p_{1-s}\left(\Sigma_{s}, e\right)\right)\right||h(s)|$. Hence using (2.2) and (2.11) as above one shows for sufficiently large constants $K$ and $C$ that

$$
L \leq K \limsup _{s \uparrow 1} \nu_{e}\left(\left|\nabla \ln \left(p_{1-s}\left(\Sigma_{s}, e\right)\right) \| h(s)\right|\right) \leq C \limsup _{s \uparrow 1}[|h(s)| / \sqrt{1-s}] .
$$

Since by assumption $h(1)=0$ and $h$ is $C^{1}$, the last limit is clearly 0 and hence $L=0$.

\section{ACKNOWLEDGMENTS}

It is a pleasure to acknowledge M. Cranston, M. Gage, W. Kendall, P. Li., and A. Setti for their efforts in trying to pointing me to the correct source for the gradient estimate used in this paper. I would especially like to thank R. Hamilton for sharing his gradient estimate results in [23] with me. It is also a pleasure to thank P. Fitzsimmons, R. Williams, and M. Yor for a number of useful conversations dealing with probability theory and " $h$-transforms."

\section{REFERENCES}

1. H. Airault and P. Malliavin, Integration on loop group. II; Heat equation for the Wiener measure, J. Funct. Anal. 112 (1992), 71-109.

2. S. Albeverio and R. Hoegh-Krohn, The energy representation of Sobolev Lie groups, Compositio Math. 36 (1978), 37-52.

3. Jean-Michel Bismut, Mecanique aleatoire, Lecture Notes in Math., vol. 866 (A. Dold and B. Eckmann, eds.), Springer-Verlag, Berlin, Heidelberg, New York, 1981.

4. L_Large deviations and the Malliavin calculus, Birkhäuser, Boston and Basel, 1984.

5. P. Brémaud and M. Yor, Changes of filtrations and of probability measures, $\mathrm{Z}$. Wahrsch. verw. Gebiete 45 (1978) 269-295.

6. R. H. Cameron, The first variation of an indefinite Wiener integral, Proc. Amer. Math. Soc. 2 (1951), 914-924.

7. R. H. Cameron and W. T. Martin, Transformations of Wiener integrals under translations, Ann. of Math. (2) 45 (1944), 386-386.

8. Transformations of Wiener integrals under a general class of linear transformations, Trans. Amer. Math. Soc. 58 (1945), 184-219.

9. $ـ$ The transformation of Wiener integrals by non-linear transformations, Trans. Amer. Math. Soc. 66 (1949), 253-283.

10. J. Cheeger and S. T. Yau, A lower bound for the heat kernel, Comm. Pure Appl. Math. 34 (1981), 465-480.

11. E. B. Davies, Heat kernels and spectral theory, Cambridge Univ. Press, Cambridge and New York, 1990.

12. J. L. Doob, Classical potential theory and its probabilistic counterpart, Springer-Verlag, Berlin, Heidelberg, New York, 1983.

13. B. Driver, A Cameron-Martin type quasi-invariance theorem for Brownian motion on a compact Riemannian manifold, J. Funct. Anal. 110 (1992), 272-376.

14. Michel Emery, Stochastic calculus in manifolds, Springer-Verlag, Berlin, Heidelberg, New York, 1989.

15. Epperson and Lohrenz, Brownian motion on the path space of a compact Lie group, Rice Univ. preprint, 1991.

16. I. B. Frenkel, Orbital theory for affine Lie algebras, Invent. Math. 77 (1984), 301-352. 
17. I. V. Girsanov, On transforming a certain class of stochastic processes by absolutely continuous substitution of measures, Theory Probab. Appl. 5 (1960), 285-301.

18. P. Gilkey, Invariance theory, the heat equation, and the Atiyah-Singer index theorem, Publish or Perish, Wilmington, DE, 1984.

19. L. Gross, Integration and nonlinear transformations in Hilbert space, Trans. Amer. Math. Soc. 94 (1960), 404-440.

20. __ Abstract Wiener spaces, Proc. 5th Berkeley Sympos. Math. Stat. Prob. 2, 1965, pp. $31-42$.

21. Logarithmic Sobolev inequalities for the heat kernel of a Lie group, White Noise Analysis : Mathematics and Applications, (T. Hida et al., eds.) World Scientific, Singapore and Teaneck, NJ, 1990, pp. 108-130.

22. __ Logarithmic Sobolev inequalities on loop groups, J. Funct. Anal. 102 (1991), 268313.

23. Richard Hamilton, A matrix Harnack estimate for the heat equation, Comm. Anal. \& Geom. 1 (1993), 113-126.

24. N. Ikeda and S. Watanabe, Stochastic differential equations and diffusion processes, NorthHolland, Amsterdam, 1981.

25. J. D. S. Jones and R. Leandre, $L^{p}$-Chen forms on loop spaces, Stochastic Analysis, Barlow and Bingham, eds., Cambridge Univ. Press, Cambridge and New York, 1991, pp. 103-162.

26. S. Kobayashi and K. Nomizu, Foundations of differential geometry, Vol. 1, Interscience, New York, 1963.

27. H.-H. Kuo, Integration theory on infinite-dimensional manifolds, Trans. Amer. Math. Soc. 159 (1971) 57-78.

28. $\ldots$, Diffusion and Brownian motion on infinite dimensional manifolds, Trans. Amer. Math. Soc. 169 (1972) 439-457.

29. —_ Gaussian measures in Banach spaces, Lecture Notes in Math., vol. 463, SpringerVerlag, Berlin and New York, 1975.

30. S. Kusuoka, The nonlinear transformation of Gaussian measure on Banach space and its absolute continuity. I, J. Fac. Sci. Univ. Tokyo Sect. IA Math. 29 (1982), 567-597.

31. _ On the foundations of Wiener-Riemannian manifolds, Stochastic Analysis, Path Integration and Dynamics: Emanations from "Summer Stochastics" Warwick 1987 (K. D. Elworthy and J.-C. Zambrini, eds.), Wiley, New York, 1989, pp. 130-165.

32. __ Analysis on Wiener spaces. I: Nonlinear maps, R.I.M.S. report 670, 1989.

33. _ Analysis on Wiener spaces. II: Differential forms, R.I.M.S. report 705, 1990.

34. P. Li and S. T. Yau, On the parabolic kernel of the Schrödinger operator, Acta Math. 156 (1986), 153-201.

35. M-P. Malliavin, and M. Malliavin, Integration on loop groups. I: Quasi invariant measures, J. Funct. Anal. 93 (1990), 207-237.

36. , Integration on loop group. III. Asymptotic Peter-Weyl orthogonality, J. Func. Anal. 108 (1992), 13-46.

37. _ La representation reguliere des groupes de lacets, Proc. Conf. Trientenaire 1990, Hamburg Math. Sem., in the Third Centenary Anniversary of Hamburg University.

38. _ An infinitesimally quasi invariant measure on the group of diffeomorphisms of the circle, Proc. Hashibara Forum (M. Kashiwara and T. Miwa, eds.), 1991.

39. P. Malliavin, Naturality of quasi-invariance of some measures, Proc. Lisbonne Conf., Birkhäuser (A. B. Cruzerio, ed.).

40. —_ Hypoellipticity in infinite dimension, Diffusion Process and Related Problems in Analysis, Vol I. (M. A. Pinsky, ed.), Birkhäuser, 1991.

41. _ Diffusion on the loops, Proc. Conf. in the Honour of Antoni Zygmund, Chicago 1981-1983 (W. Beckner and A. Calderon, eds.), Vol. 2, Wadsworth Internat. Group, 1983, pp. 764-782.

42. G. Maruyama, Notes on Wiener integrals, Kodai Math. Sem. Rep. 3 (1950), 41-44. 
43. J. R. Norris, Path integral formulae for heat kernels and their derivatives, Probab. Theory Related Fields 94 (1993), 525-541.

44. P. Protter, Stochastic integration and differential equations; a new approach, Springer-Verlag, Berlin, Heidelberg, New York, 1990.

45. R. Ramer, On nonlinear transformations of Gaussian measures, J. Funct. Anal. 15 (1974), 166-187.

46. D. Revuz and M. Yor, Continuous martingales and Brownian motion, Springer-Verlag, Berlin, Heidelberg, New York, 1991.

47. John Roe, Elliptic operators, topology, and asymptotic methods, Pitman Res. Notes Math. Ser., no. 179, Wiley, New York, 1988.

48. L. C. G. Rogers and D. Williams, Diffusions, Markov processes, and martingales. Vol. 2 : Ito calculus, Wiley, New York, 1987.

49. A. Setti, Eigenvalue estimates for the Laplacian with lower order terms on a complete Riemannian manifold, Cornell Univ. preprint, 1990.

50. C. Stricker and M. Yor, Calcul stochastique dépandant d'un parametre, Z. Wahrsch. Verw. Gebiete 45 (1978), 109-103.

51. D. W. Stroock, Lectures on topics in stochastic differential equations, Lectures given at Tata Institute of Fundamental Research, Bombay, 1982, Springer-Verlag, Berlin, Heidelberg, New York, 1982.

52. __ Lectures on stochastic analysis: diffusion theory, Cambridge Univ. Press, Cambridge and New York, 1987.

53. D. W. Stroock and Varahdhan, Multidimensional diffusion processes, Springer-Verlag, Berlin, Heidelberg, New York, 1979.

54. N. Th. Varopoulos, Small time Gaussian estimates of heat diffusion kernels. I: The semigroup technique, Bull. Sci. Math. (2) 113 (1989), 253-277.

55. __ Small time Gaussian estimates of heat diffusion kernels. II: The theory of large deviations, J. Funct. Anal. 93 (1990), 1-33.

56. Marc Yor, Grossissement de filtrations et absolue continuite de noyaux, Lecture Notes in Math, vol. 1118 (Th. Jeulin and M. Yor, eds.), Springer-Verlag, Berlin and New York, 1985.

Department of Mathematics, University of California at San Diego, la Jolla, CaliFORNIA 92093-0112

E-mail address: driver@euclid.ucsd.edu 